\title{
Commentary: Ginkgo Biloba for Mild Cognitive Impairment and Alzheimer's Disease: A Systematic Review and Meta-Analysis of Randomized Controlled Trials
}

\author{
Guoyan Yang ${ }^{1,2}$, Dennis Chang' and Jianping Liu' ${ }^{2 *}$ \\ 'National Institute of Complementary Medicine, Western Sydney University, NSW 2751, Australia \\ ${ }^{2}$ Center for Evidence-Based Chinese Medicine, Beijing University of Chinese Medicine, Beijing 100029, China
}

Article Info

\section{Article Notes}

Received: October 20, 2016

Accepted: November 15, 2016

\section{${ }^{*}$ Correspondence:}

Dr. Jianping Liu, Center for Evidence-Based Chinese Medicine, Beijing University of Chinese Medicine, Beijing 100029, China, Tel: +86-010-6428-6760; Fax: +86-010-6428-6760;

E-mail: Liujp@bucm.edu.cn

(c) $2016 \mathrm{Liu} \mathrm{J}$. This article is distributed under the terms of the Creative Commons Attribution 4.0 International License

\section{Keywords:}

DBS

addiction

ethics

patient autonomy

informed consent

clinical trials

\section{ABSTRACT}

Background: Ginkgo biloba is a natural medicine used for cognitive impairment and Alzheimer's disease.

Methods: This is a commentary on a systematic review that we conducted to evaluate the effectives and safety of Ginkgo biloba in treating mild cognitive impairment or Alzheimer's disease.

Results: 21 articles with 2608 patients met the inclusion criteria. The general methodological quality of included studies was moderate to poor. Compared with conventional medicine alone, Ginkgo biboba in combination with conventional medicine was superior in improving Mini-Mental State Examination (MMSE) scores at 24 weeks for patients with Alzheimer's disease (MD 2.39, 95\% $\mathrm{Cl} 1.28$ to $3.50, \mathrm{P}<0.0001$ ) and mild cognitive impairment (MD $1.90,95 \% \mathrm{Cl} 1.41$ to $2.39, \mathrm{P}<0.00001$ ), and Activity of Daily Living (ADL) scores at 24 weeks for Alzheimer's disease (MD $-3.72,95 \% \mathrm{Cl}-5.68$ to $-1.76, \mathrm{P}=0.0002$ ). When compared with placebo or conventional medicine in individual trials, Ginkgo biboba demonstrated similar but inconsistent findings. Adverse events were mild.

Conclusion: Ginkgo biloba is potentially beneficial for the improvement of cognitive function, activities of daily living, and global clinical assessment in patients with mild cognitive impairment or Alzheimer's disease. However, due to limited sample size, inconsistent findings and methodological quality of included trials, more research are warranted to confirm the effectiveness and safety of Ginkgo biloba in treating mild cognitive impairment and Alzheimer's disease.

\section{Introduction}

Mild cognitive impairment (MCI), characterized by a slight but noticeable decline in cognitive function ${ }^{1}$, is regarded as a symptomatic stage before progression to dementia, especially Alzheimer's disease $(\mathrm{AD})^{2}$. $\mathrm{AD}$, a common type of dementia, is characterized by a progressive decline in cognition, activities of daily living with behavioral disturbances ${ }^{3}$. No current medications can cure MCI and AD. Some symptomatic treatments with conventional medications may be beneficial in lessening memory loss and confusion; however, these medications have side effects such as nausea, vomiting, and loss of appetite ${ }^{4}$.

In recent years, an increasing number of journal articles paid attention to natural medicines such as Ginkgo biloba. It is encouraging that many clinical trials ${ }^{5-10}$ and reviews ${ }^{11-15}$ demonstrate that Ginkgo 
biloba could stop or slow cognitive deterioration, functional and behavioral disturbances in patients with dementia or MCI.

\section{Methods}

In an effort to evaluate the potential benefits and risks relevant to Ginkgo biloba in treating patient with MCI and $\mathrm{AD}$, we conducted a systematic review of published articles. We searched six electronic databases in English and Chinese for eligible articles. The detailed search strategy, study selection, and data extraction process are available in the published full paper ${ }^{16}$.

We included randomized control trials with at least one group involving ginkgo biloba for the treatment of AD or MCI. Participants with MCI or AD should be diagnosed with validated criteria. The key differences of diagnostic criteria between $\mathrm{AD}$ and $\mathrm{MCI}$ were as follows: (1) AD can be diagnosed only if at least two domains (including memory) demonstrating cognitive impairment, while MCI can be diagnosed if there is impairment in memory; (2) AD can be diagnosed only if cognitive impairment is so severe that the ability to perform activities of daily livings is interfered, while for MCI, there is just a slight cognitive decline and functional disturbance ${ }^{17}$. We excluded trials involving participants with other types of dementia.

We pooled data by meta-analyses with RevMan 5.2 software. We summarized data of binary outcomes using odds ratios (OR) with 95\% confidence intervals (CI), and data of continuous outcomes using mean difference (MD) with 95\% CI. Random effects model was applied to analyze data with substantial heterogeneity.

\section{Results}

21 articles (12 articles of $\mathrm{AD}$ and nine of $\mathrm{MCI}$ ) with 2608 patients were included in the systematic review. The duration of treatment, the measurement of outcomes, the forms of Ginkgo biloba preparation, the dosage of Ginkgo biloba, and the comparisons were not consistently reported across articles. Despite less than ideal reporting in many cases, the included articles allowed us to summarize the effectiveness and safety of Ginkgo biloba narratively and in meta-analyses.

Compared with conventional medicine alone, Ginkgo biboba in combination with conventional medicine was superior in improving cognitive function measured by MiniMental State Examination (MMSE) scores at 24 weeks for patients with AD (MD 2.39, 95\% CI 1.28 to $3.50, \mathrm{P}<0.0001$ ) and MCI (MD 1.90, 95\% CI 1.41 to $2.39, \mathrm{P}<0.00001$ ), and Activity of Daily Living (ADL) scores at 24 weeks for AD (MD -3.72, 95\% CI -5.68 to $-1.76, \mathrm{P}=0.0002$ ). When compared with placebo or conventional medicine alone, Ginkgo biboba demonstrated similar but inconsistent findings within individual articles or among articles.
When compared with placebo or conventional medicine alone, individual trials and meta-analysis favored Ginkgo biboba in improving activities of daily living, global clinical assessment. However, no significant differences were also reported in individual articles when measured by various scales in different durations. Only one trial comparing Ginkgo biloba with placebo for AD reported quality of life and favored Ginkgo biloba.

Half of the 21 articles reported safety information. Most adverse events were considered of mild intensity, such as nausea, dizziness, headache, etc. A meta-analysis showed there was no significant difference between groups in adverse events.

\section{Limitations}

In this systematic review, the general methodological quality of included trials was limited. Though potential benefits of Ginkgo biloba for MCI and AD were reported, inconsistent results within or among individual articles were also identified.

We documented a variety of scales in included articles evaluating the effects of Ginkgo biloba for MCI or AD on cognitive function, activities of daily living, and global clinical changes, which increased the difficulty when we pooled data. The variety indicates that there is no gold standard measurement for these outcomes. For quality of life, only one article evaluated this important outcome relevant to patients. Further researchers are recommended to report these important outcomes measured by reliable and validated scales.

Though most adverse events were reported as mild and the rates of adverse events were similar for Ginkgo biloba and placebo, only half of the included 21 randomized controlled trials reported safety information. Previous reviews $^{18-19}$, preclinical studies ${ }^{20-21}$ and clinical studies ${ }^{22-23}$ also identified the relative safety of Ginkgo biloba for the treatment of MCI and dementia. Therefore, Ginkgo biloba for MCI or AD is relatively safe. However, in most included articles the duration of treatment was 24 weeks, so longterm safety and potential effects of Ginkgo biloba for AD or MCI is not clear.

The dose of Ginkgo biloba varied among included articles. The possible reason is that there are different preparations of Ginkgo biloba including standardized Ginkgo biloba extract EGb 761 and other forms such as tablet, pill and injection. We suggest future researchers report the preparations/forms of Ginkgo biloba as well as definite descriptions of active ingredients (e.g standardized Ginkgo biloba extract EGb 761), so readers who are interested in Ginkgo biloba could understand the intervention and might tailor it to their particular context. 


\section{Conclusion}

The most useful result of the completed systematic review is that most meta-analyses and individual articles demonstrated that Ginkgo biboba had potential benefits in improving cognitive function, activities of daily living, and global clinical assessment for the treatment of MCI and AD when measured by various scales in different durations.

However, there is insufficient evidence supporting us to draw confirmative conclusions, considering the limited sample size, inconsistent results among individual trials, and limited methodological quality of included articles. We encourage more publication of high-quality research and experiences about Ginkgo biloba, so the evidence of Ginkgo biloba for MCI and AD can become clearer and more valuable for people with MCI or AD.

\section{Acknowledgements}

The completed systematic review is supported by the Program of Innovative Research Team of Beijing University of Chinese Medicine (2011-CXTD-09).

\section{References}

1. Kenneth ML, Deborah AL. The diagnosis and management of mild cognitive impairment: a clinical review. JAMA. 2014; 312(23): 25512561.

2. Peterson RC, Roberts RO, Knopman DS, Boeve BF, Geda YE, Ivnik RJ, et al. Mild cognitive impairment: ten years later. Neurol. 2009; 66(12): 1447-1455.

3. Hanns H, Gabriele N. The discovery of Alzheimer's disease. Dialogues Clin Neurosci. 2003; 51: 101-108.

4. Alzheimer's Association. FDA-Approved Treatment for Alzheimer's. 2016. Available from: www.alz.org. [Accessed 25th September, 2016].

5. Ihl R, Tribanek M, Bachinskaya N. Efficacy and tolerability of a once daily formulation of Ginkgo biloba extract EGb 761(R) in Alzheimer's disease and vascular dementia: results from a randomized controlled trial. Pharmacopsychiatry. 2012; 45(2): 41-46.

6. Le Bars PL, Katz MM, Berman N, Itil TM, Freedman AM, Schatzberg AF. A placebo-controlled, doubleblind, randomized trial of an extract of ginkgo biloba for dementia. JAMA. 1997; 278(16): 1327-1332.

7. Maurer K, Ihl R, Dierks T, Froloch L. Clinical efficacy of Ginkgo biloba special extract Egb 761 in dementia of the Alzheimer's type. J Psychiat Res. 1997; 31(6): 645-655.

8. Mazza M, Capuano A, Bria P, Mazza S. Ginkgo biloba and donepezil: A comparison in the treatment of Alzheimer's dementia in a randomized placebo-controlled double-blind study. European J Neurol. 2006; 13(9): 981-985.

9. Nasab NM, Bahrammi MA, Nikpour MRA, Rahim F, Naghibi SN. Efficacy of rivastigmine in comparison to ginkgo for treating Alzheimer's dementia. J Pak med Assoc. 2012; 62(7): 677-680.

10. Schneider LS, Dekosky ST, Parlow MR, Tariot PN, Hoerr R, Kieser M. A randomized, doubleblind, placebo-controlled trial of two doses of ginkgo biloba extract in dementia of the Alzheimer's type. Current Alzheimer Research. 2005; 2(5): 541-551.

11. Ihl R. Effects of Ginkgo biloba extract Egb761 in dementia with neuropsychiatric features: review of recently completed randomised, controlled trials. Int J Psychiatry Clin Pract. 2013; 17(Suppl 1): 8-14.

12. Tan MS, Yu JT, Tan CC, Wang HF, Meng XF, Wang C, Jiang T, Zhu XC, Tan L. Efficacy and adverse effects of Ginkgo biloba for cognitive impairment and dementia: a systematic review and meta-analysis. J Alzhermers Dis. 2014; 589-603.

13. Birks J, Evans JG. Ginkgo biloba for cognitive impairment and dementia. Cochrane Database of Systematic Reviews. 2009; 1. Art. No.: CD003120. DOI: 10.1002/14651858.CD003120.pub3.

14. JanBen IM, Sturtz S, Skipka G, Zentner A, Garrido MV, Busse R. Ginkgo biloba in Alzheimer's disease: a systematic review. Wien Med Wochenschr. 2010; 160(21-22): 539-546.

15. Yang M, Xu DD, Zhang Y, Liu X, Hoeven R, Cho WC. A systematic review on natural medicines for the prevention and treatment of Alzheimer's disease with meta-analyses of intervention effect of Ginkgo. Am J Chin Med. 2014; 42(3): 505-521.

16. Yang G, Wang Y, Sun J, Zhang K, Liu J. Ginkgo Biloba for Mild Cognitive Impairment and Alzheimer's Disease: A Systematic Review and MetaAnalysis of Randomized Controlled Trials. Curr Top Med Chem. 2016; 16(5):520-528.

17. Lee SJ, Ritchie CS, Yaffe K, Cenzer IS, Barnes DE. A clinical index to predict progression from mild cognitive impairment to dementia due to Alzheimer's disease. PLoS One. 2014; 9(12): e113535.

18. Hashiguchi M, Ohta Y, Shimizu M, Maruyama J, Mochizuki M. Metaanalysis of the efficacy and safety of Ginkgo biloba extract for the treatment of dementia. J Pharm Health Care Sci. 2015; 1:14.

19. Mahadevan S, Park Y. Multifaceted therapeutic benefits of Ginkgo biloba L.: chemistry, efficacy, safety and uses. J Food Sci. 2008; 73(1): R14-19.

20. Heinonen T, Gaus W. Cross matching observations on toxicological and clinical data for the assessment of tolerability and safety of Ginkgo biloba leaf extract. Toxicology. 2015; 327: 95-115.

21. Lingli M, Junping K, Danni Z, Boyang Y. Comparison of neuroprotective effects of Flavonoids, Terpenoids, and their combinations from Ginkgo biloba on Ischemia-Reperfusion-Injured mice. Pharmaceutical Biology. 2007; 45(9): 728-733.

22. Gavrilova SI, Preuss UW, Wong JW, Hoerr R, Kaschel R, Bachinskaya N; GIMCIPlus Study Group. Efficacy and safety of Ginkgo biloba extract EGb 761 in mild cognitive impairment with neuropsychiatric symptoms: a randomized, placebo-controlled, double-blind, multicenter trial. 2014; 29(10):1087-1095.

23. Herrschaft H, Nacu A, Likhachev S, Sholomov I, Hoerr R, Schlaefke S. Ginkgo biloba extract EGb 761 in dementia with neuropsychiatric features: a randomised, placebo-controlled trial to confirm the efficacy and safety of a daily dose of $240 \mathrm{mg}$. 2012; 46(6):716-723. 